\title{
A detecting and identifying method for two stationary human targets using single-channel ultra-wideband radar
}

\author{
Yang Zhang ${ }^{\dagger}$, Teng Jiao ${ }^{\dagger}$, Xijing Jing, Zhao Li, Sheng Li, Xiao Yu, Hao Lv, Zhu Zhang and Jianqi Wang*
}

\begin{abstract}
A novel technique for single-channel ultra-wideband (UWB) radar to detect and identify two stationary human subjects in different positions is proposed in this article. To detect two stationary human targets accurately and get their life parameters precisely, space-frequency analysis is performed upon the preprocessed data in horizontal dimension and then a non-linear threshold is calculated for automatic identification. Experiments are conducted to evaluate the effectiveness of the proposed technique. In 61 data sets with two stationary human subjects, the accuracy of identification reaches $72 \%$. The experimental results demonstrate the validity of our approach to detect and identify two stationary human subjects using single-channel UWB radar system. This technique may serve as a basis for further studies on a two-dimensional locator for multi-stationary human subjects via multi-channel UWB radar.
\end{abstract}

Keywords: Multi-stationary subjects, Space-frequency analysis, UWB, Detection

\section{Introduction}

Life-detection radar is a novel kind of radar that combines the technology of radar and biomedical engineering. It can detect vital signals (such as respiration, heartbeat, jiggle, etc.) within a certain distance through nonmetallic substances (such as brick walls, rubble, etc.) without using electrodes, and is thus considered a powerful tool for a variety of civilian, law enforcement, and military applications. Accurate identification and location can allow the police force to get a precise description of a hostage crisis in a building, or allow firefighters to locate people trapped inside a burning structure. Moreover, lifedetection radar has recently been sought out in other rescue missions, such as searching for earthquake and avalanche survivors.

Life-detection radar was developed originally in a Continuous Wave (CW) radar system [1-4] since 1980s. However, the application of CW radar was limited because the reflected wave could not provide range and azimuth information. To obtain this essential information,

\footnotetext{
* Correspondence: wangjq@fmmu.edu.cn

${ }^{\dagger}$ Equal contributors

School of Biomedical Engineering, The Fourth Military Medical University, Xi'an 710032, China
}

the ultra-wideband (UWB) life-detection radar is now becoming a popular alternative due to its high range resolution and target-identifying capability. Some research groups study on identifying and locating moving multitargets with UWB life-detection radar [5-8]. And shorttime Fourier transform (STFT) is also applied in the detection algorithm $[9,10]$, But there are only a few reports on detecting and identifying multi-stationary human targets. Since the chest-wall motion due to the respiration is very small, existing UWB radars for the application usually operate at high center frequencies (above $600 \mathrm{MHz}$ ) [11-14], and show limited penetration capability in experiments. This leads to low detection capability and difficult to extract multi-stationary human life characteristic from the signals. Therefore, multistationary human-targets identification and localization, a key problem limiting the application of life-detection radar, has not yet been resolved $[15,16]$.

In this article, a new UWB radar operating at a center frequency of $400 \mathrm{MHz}$ is developed to improve the detection capability through nonmetallic substances, and a method combined several signal processing techniques for two stationary human targets detection is proposed. First of all, in order to enhance the stationary human life

\section{目 Springer}


characteristic and improve the signal-to-noise ratio (SNR) of the echo, the filtering and moving average subtraction are implemented. Then, space-frequency analysis that time is changed by space in a time-frequency function and a non-linear threshold are used to identify two stationary human subjects in different distances. Finally, the experimental results and discussion are presented.

\section{Methods}

\section{Description of the system}

The schematic diagram of the single-channel UWB radar system is illustrated in Figure 1. The oscillator generates a narrow pulse signal with a center frequency of $400 \mathrm{MHz}$ that is controlled by a pulse coder. The output of the oscillator is fed through an electromagnetic pulse generator to the transmitting antenna. The bowtie dipole antenna transmits vertically polarized pulses with a peak power of about $5 \mathrm{~W}$. The echo is received by the receiving antenna and then sent to the sample integrator, and the signal out from the oscillator generates range gates through a delay circuit and a range gates generator. The echo signal is sampled according to the range gates and is accumulated by an integral circuit, which next leads to the extraction of the weak low-frequency signal. This signal is amplified and filtered by a signal processor and passed through a high-speed Analog/Digital (A/D) converter before reaching the Digital Signal Processor (DSP) for further processing. Finally, the distance of each human target is computed by DSP and sent to a PC terminal to display the detecting result via Bluetooth.

\section{Decomposition and synthesis of the signal}

The basic flowchart of the signal processing is shown in Figure 2. The amount of data is too large for real-time computation, so that the signal out from the A/D converter should be integrated by sliding the window first to cut down the length of the data. Great computational load is avoided by the integration, but the SNR of signal is not decreased. The echo $s(t, d)$ is a function of two parameters: the delay or range (distance) and the time.

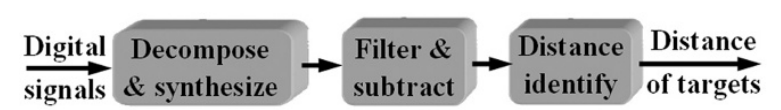

Figure 2 The basic flowchart of signal processing.

The echo is then decomposed and synthesized in the time and space domain. Thus, Time Signal of echo $x(t)$ is defined as $x(t)=s(t, d)$ for a fix distance, and Distance Signal $y(d)=s(t, d)$ for a fix time.

\section{Improving SNR by filtering and moving average subtraction}

The Time Signals of echo $x(k)$ are filtered by a 160 -order low-pass Hamming window-based filter with a cut-off frequency of $0.5 \mathrm{~Hz}$ to restrain high-frequency noise and keep the respiration signal of human. A moving average subtraction is then applied to remove the background clutter of the echo, which can enhance the stationary human life characteristic of the signals and improve the SNR. The formula of moving average subtraction is as follows:

$$
X(n)=x(n)-\frac{\sum_{k=n-m}^{k=n-1} x(k)}{m},
$$

where $X$ is the output signal, $x$ is the input signal, $m$ is the width of moving window, and $n$ is the sequence number of the Time Signal of echo.

\section{Distance identification by space-frequency analysis}

The signal (time window is $60 \mathrm{~ns}$ : range $0-9 \mathrm{~m}$ ) from the subtraction is divided equally into 26 segments. The number of segments is selected according to the range resolution needed. The distance denoted by each segment is about $0.36 \mathrm{~m}$. The first four segments are cut out to remove the interference caused by the direct-coupling arrival (echo directly from the transmitting antenna to the receiving one). Then, every amplitude of $y(d)$ in each segment from the 5 th to 26 th is summed respectively. As

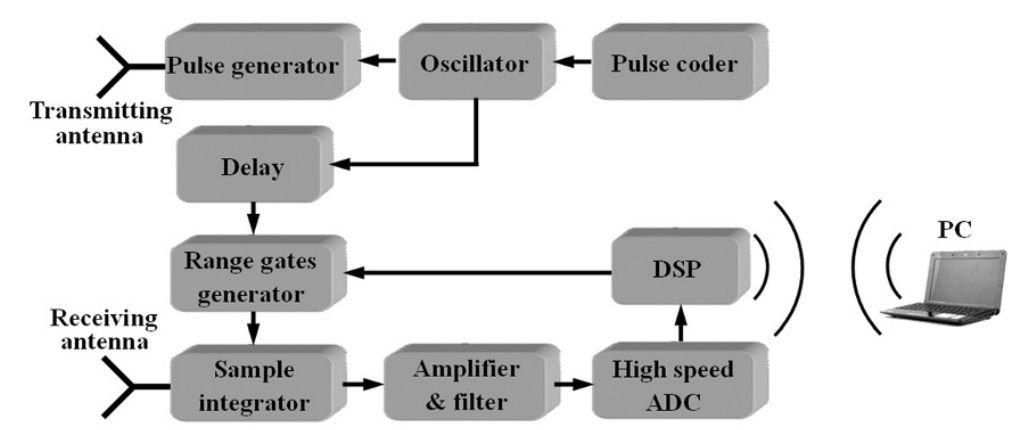

Figure 1 Schematic of the UWB life-detection radar system. 


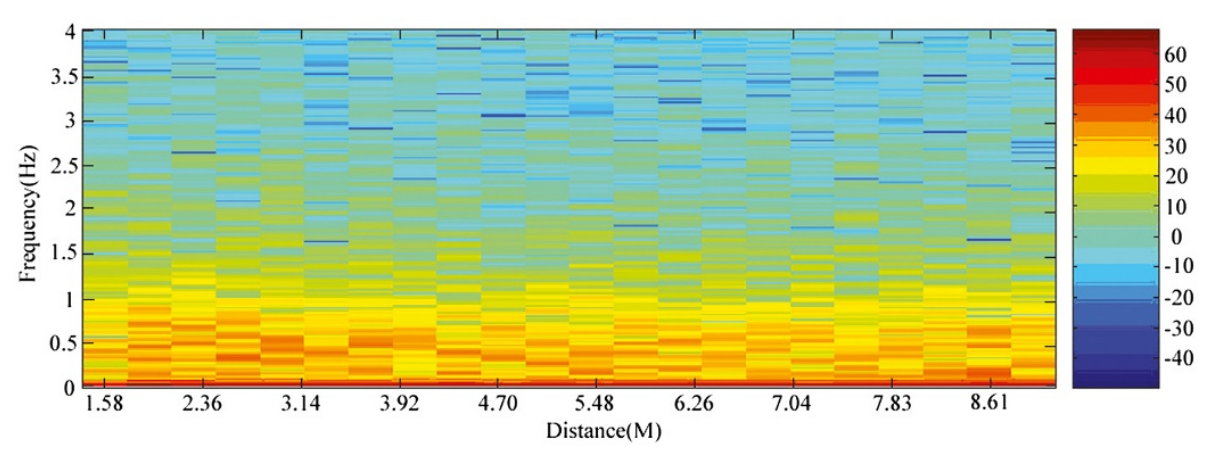

Figure 3 STFT spectrogram of a no-subject (free space).

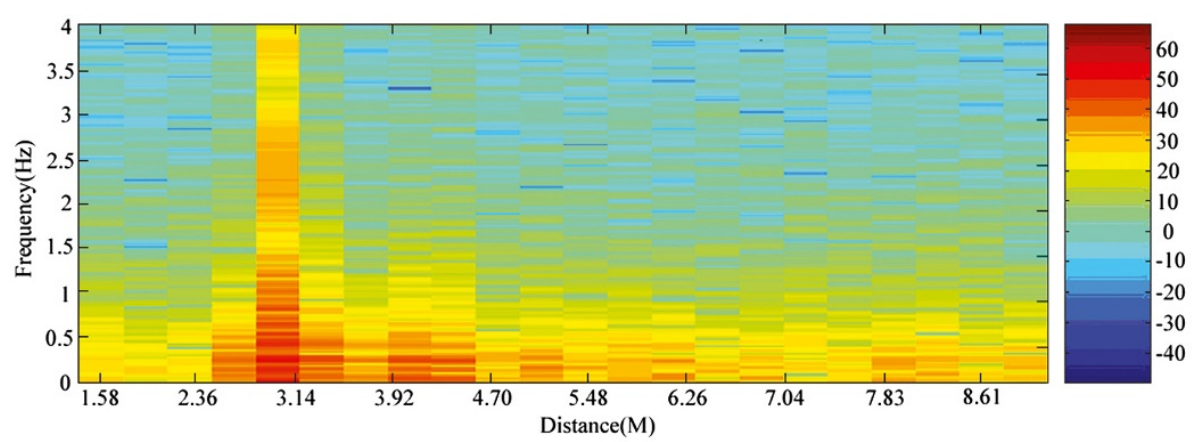

Figure 4 STFT spectrogram of one stationary human subject in $2.5 \mathrm{~m}$ (free space).
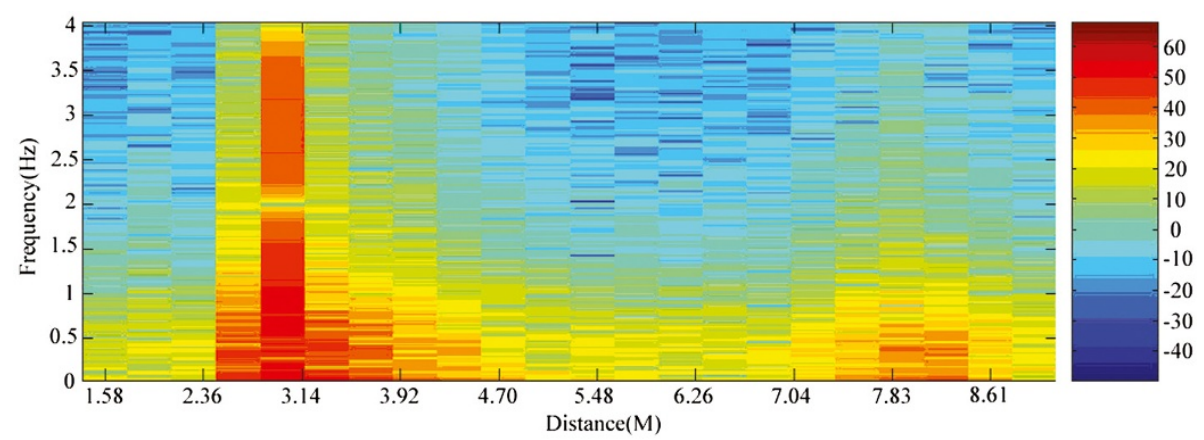

Figure 5 STFT spectrogram of two stationary human subjects in 2.5 and $7.5 \mathrm{~m}$, respectively (free space). 
Table 1 Experimental results of a 23-set data on no-subject

\begin{tabular}{lccccc}
\hline & True & False & Missing & Error & Total \\
\hline Number & 21 & 2 & 0 & 0 & 23 \\
Percentage & $91 \%$ & $9 \%$ & $0 \%$ & $0 \%$ & $100 \%$ \\
\hline
\end{tabular}

a result, a new Distance Signal is composed by these 22 sums.

In the light of the time resolution needed in practical detection, the data length is selected as $10 \mathrm{~s}$. So in the time domain the collected data forms 22 new Time Signals of echo which last for 10s. Finally, according to the range sequence, these new Time Signals of echo are connected and regarded as the Input Signal.

STFT is performed to the Input Signal, and the window width used is equal to the length of the new Time Signal of echo. The number of Fourier Transform points is 1024, thus, the STFT can be expressed as:

$$
\operatorname{STFT}(t, w)=\int S(\tau) \gamma(\tau-t) \exp (-j w \tau) d \tau
$$

where $S(\tau)$ is the Input Signal, $\gamma(t)$ is the window function.

The typical STFT of stationary human signals is respectively shown in Figures 3, 4 and 5.

Threshold of estimation: The result of space-frequency transformation denotes a three-dimensional relation. Two coordinates represent the range and the frequency respectively, and the colors of the spectrogram represent the signal power amplitude. An appropriate adaptive non-linear threshold is chosen to distinguish the multistationary subjects. If the power amplitude in the frequency range of human respiration at a certain distance is obviously higher than that at other distances, and exceeds the threshold simultaneously, then the result is judged such that there is a stationary human in this distance (one-dimensional distance). If more than one highpower spectral peak appears at different distances, then many stationary human targets are assumed in the corresponding distances. And these target distances are recorded; thus, the soft threshold can be expressed as:

$$
E_{n}>|\rho| E_{\text {mean }}
$$

where $E_{\mathrm{n}}$ is the power of spectral peak, and $E_{\text {mean }}$ is the mean power of the 22 segment signals. $|\rho|$ is determined

Table 2 Experimental results of a 30 -set data on single human subject

\begin{tabular}{lccccc}
\hline & True & False & Missing & Error & Total \\
\hline Number & 19 & 6 & 3 & 2 & 30 \\
Percentage & $63 \%$ & $20 \%$ & $10 \%$ & $7 \%$ & $100 \%$ \\
\hline
\end{tabular}

Table 3 Experimental results of a 61-set data on two human subjects

\begin{tabular}{cccccc}
\hline & True & False & Missing & Error & Total \\
\hline Number & 44 & 2 & 6 & 9 & 61 \\
Percentage & $72 \%$ & $3 \%$ & $10 \%$ & $15 \%$ & $100 \%$ \\
\hline
\end{tabular}

according to the distance from radar because of the attenuation of the power along with the increasing distance. For human target detection, UWB radar's performance in terms of power is governed by

$$
\frac{P_{r}}{P_{t}}=\frac{G_{t} G_{r} \xi_{t} \xi_{r} \lambda \sigma}{(4 \pi)^{3} d^{4}} e^{-4 \alpha d / 8.686},
$$

where $P_{t}$ and $P_{r}$ are respectively the transmitting power and the receiving power, $G_{t}$ and $G_{r}$ are gains of the transmitting and receiving antennas, $\zeta_{t}$ and $\zeta_{r}$ are the transmitting and receiving coupling efficiencies, $\lambda$ is propagation wavelength of frequency components within the bandwidth of the radar, $\sigma$ is radar cross section of target's chest, $\alpha$ is attenuation coefficient of medium (wall or air) and $d$ is the range from the target to the UWB radar. According to the Equation (4), the formula of $|\rho|$ is

$$
|\rho|=4 \cdot e^{\frac{-d}{9}}
$$

where $d$ is the distance from radar in meters.

\section{Results}

Ten healthy volunteers (six males and four females) ranging from 20 to 37 years old, with a mean age of 27.9 $(\mathrm{SD}=5.8)$, participated in the experiments. All of the experiments were conducted in terms of the consent form signed by the volunteers according to the Declaration of Helsinki (BMJ 1991; 302:1194). The distances between radar and human subject ranged from 1 to $9 \mathrm{~m}$. The volunteers stood still in free space (no barrier between radar and human subject) during radar echo signals acquisition, and all the recorded signals were processed by the method described above.

The results were divided into four types, namely true, missing, false, and error. True is that the number and

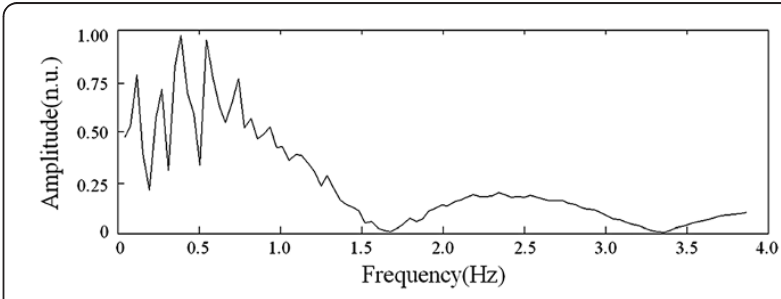

Figure 6 Frequency of the spectrogram for the body distance (2.5 m). 


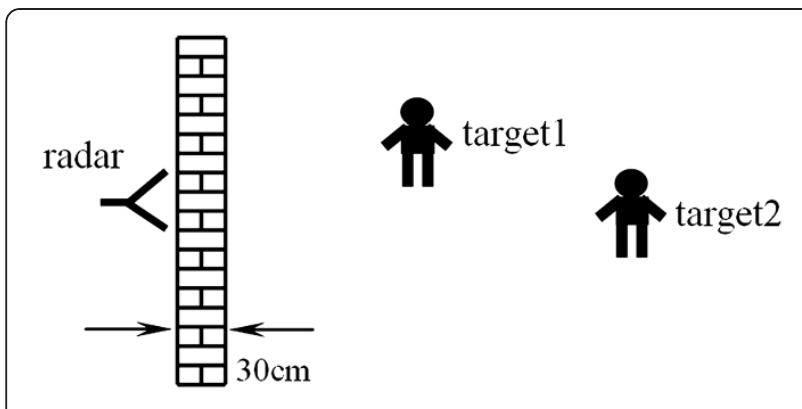

Figure 7 Schematic figure of through-wall detecting.

each distance of the human subjects are all correct, Missing is that the result shows no-subject while there is actually a human target in the detection area, False is that the result of the identification shows a subject while in a no-subject situation, and Error is that the distance of the human subject is wrong.

All data were acquired in free space scenario. Table 1 shows the results in the no-subject $(\mathrm{n}=23)$ situation.

The results in the single human subject situation $(\mathrm{n}=30)$ and the two human subjects $(\mathrm{n}=61)$ situation are shown in Table 2 and Table 3 respectively. All distances between the radar and the human subject ranged from 1 to $9 \mathrm{~m}$, and the distances between the two human subjects ranged from 1 to $6 \mathrm{~m}$. The spectrograms of the some data are shown in Figures 3, 4, and 5.

Figures 3, 4 and 5 indicate that the proposed method is quite effective in detecting and identifying one or two human subjects in free space.

The right ratio of the results in the 23-set data on nosubject is $91 \%, 63 \%$ in the 30 -set data on single human subject, and $72 \%$ in the 61 -set data on two human subjects. Tables 1, 2, and 3 indicate that the right ratio of the results in the no-subject situation is the highest while that in the single human subject situation is the lowest.

Figure 6 indicates the curve of frequency spectrogram for the body distance. Because the human target stood still in experiments, the highest peak of frequency distribution mainly dues to respiratory, to some extend, also as a result of random noise and small movement of the person. If the human subject in the detection area has small movement (swaying), the power in higher frequency band $(1 \mathrm{~Hz}-4 \mathrm{~Hz})$ of frequency spectrogram would be enhanced. If somebody is in movement (walking), the power of frequency spectrogram (in all frequency band: $0-4 \mathrm{~Hz}$ ) would be enhanced in corresponding distance where the person arrived.

The through-wall situations are also studied, where a brick wall with the thickness of $30 \mathrm{~cm}$ is placed between the radar and the human subject. The schematic diagram of detection is illustrated in Figure 7. Twenty-five sets of through-wall data were collected and processed using the method proposed in this article. The spectrograms of single human subject and two human subject signals are shown in Figures 8 and 9 respectively.

The experimental results indicate that the proposed method is also effective in detecting one or two human subjects through the brick wall. However, the effect is inferior to that in the free space due to the attenuation of radar wave through the brick wall.

\section{Discussions and conclusion}

In this article, a novel method using space-frequency analysis is proposed. This method, in which time is changed by space in time-frequency function, can analyze density of power of a non-stationary signal in the space and frequency domain simultaneously, and provide a useful estimation for power in different distances.

The initial modeling of numerical computation using single-channel UWB radar signals has been investigated. And the experimental results in this article show that the UWB radar can detect and locate two stationary human subjects by means of space-frequency analysis. The vital characteristic of human target in the signal can be extracted by the signal processing method introduced in this article. Several experiments are conducted, which prove the proposed method is more effective than the existing one in the case of detecting multi-stationary human subjects. The presented results can be regarded

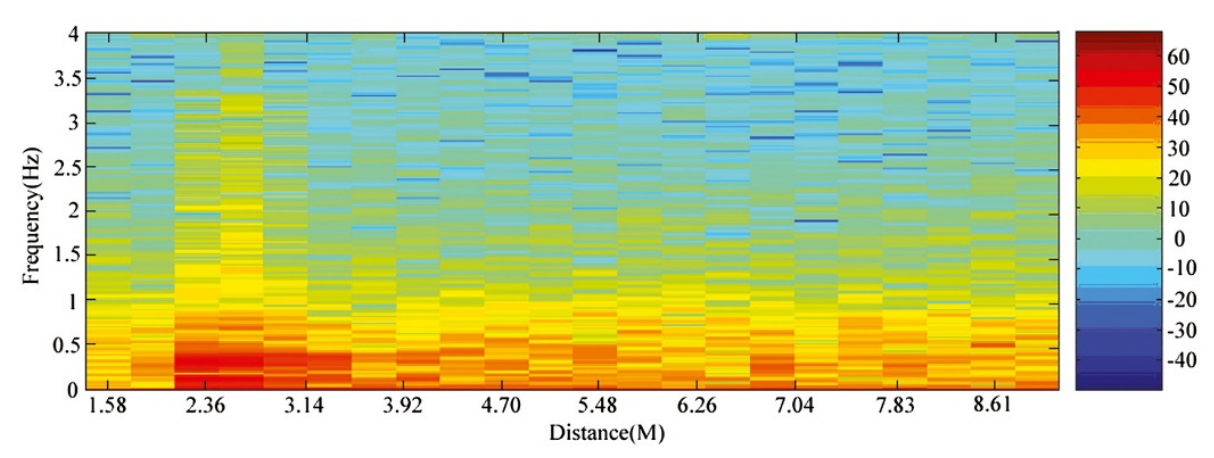

Figure 8 STFT spectrogram of one stationary human in $2.0 \mathrm{~m}$ (penetrate through brick wall that is $30 \mathrm{~cm}$ thick). 


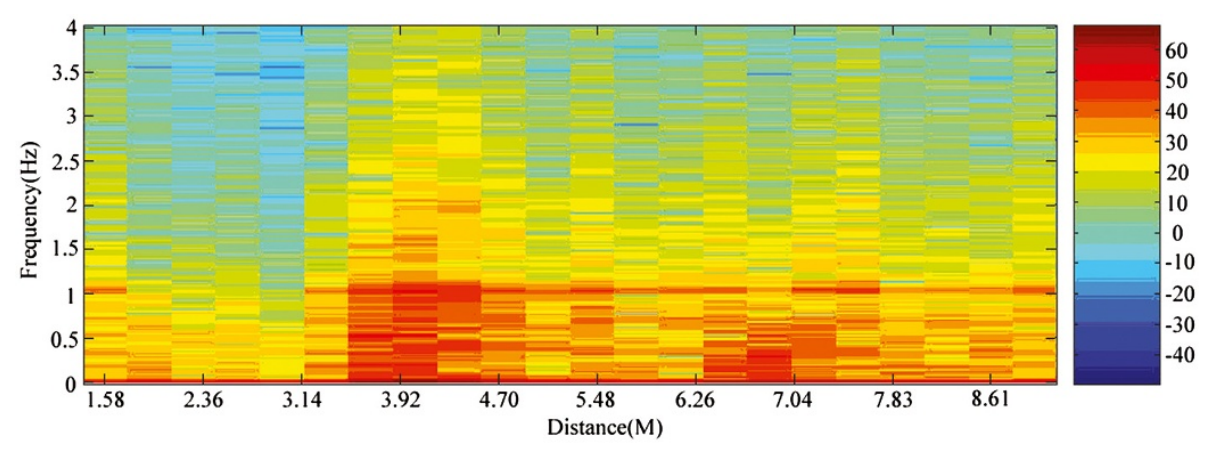

Figure 9 STFT spectrogram of two stationary human subjects in 3.0 and $6.0 \mathrm{~m}$, respectively (penetrate through brick wall that is $30 \mathrm{~cm}$ thick).

as a basis for further study on developing an appropriate two-dimensional (2-D) locator of image reconstruction models using multi-channel UWB life-detection radar.

In the experiments, the right ratio of the results in single human subject is $63 \%$, which is lower than that of the two human subjects. Analysis of the result indicates that the threshold $|\rho| \cdot E_{\text {mean }}$ is too low, which will probably lead to false alarms easily. However, if we raise the threshold $|\rho| \cdot E_{\text {mean }}$, the missing ratio of the results in the two human subjects will increase instead.

The single-channel life-detection radar prototype was used in rescue missions after Wenchuan earthquake in Sichuan Province of China, 2008. It helped rescue teams detect and locate several survivors buried under the ruins. And further studies are underway to improve the detecting sensitivity of this method and to develop a 2-D locator based on a multi-channel UWB radar system for multi-stationary-human targets.

\section{Abbreviations}

UWB: Ultra-wideband; CW: Continuous wave; SNR: Signal-to-noise ratio; DSP: Digital signal processor; STFT: Short time fourier transform; 2-D: TwOdimensional.

\section{Competing interests}

The authors declare that they have no competing interests.

\section{Authors' information}

Yang Zhang and Teng Jiao as co-first authors.

\section{Acknowledgment}

The study was carried out at the School of Biomedical Engineering, the Fourth Military Medical University, with the supportof the National Natural Science Foundation of China (NSFC) under the grants 60801059 and 60927003.

Received: 7 October 2011 Accepted: 8 September 2012 Published: 19 September 2012

\section{References}

1. S.E. Borek, B.J. Clarke, P.J. Costianes, Through-the-wall surveillance for homeland security and law enforcement. Proc SPIE 5778, 175-185 (2005)

2. P. Byung-Kwon, L. Victor, B.-L. Olga et al., Cardiopulmonary signal sensing from subject wearing body armor, inIEEE EMBS Conference, 29th edn. (, Lyon, France, 2007), pp. 366-369
3. D.R. Morgan, M.G. Zierdt, Novel signal processing techniques for Doppler radar cardiopulmonary sensing. Signal Process 89, 45-66 (2009)

4. K.M. Chen, M. Devendra, H. Wang, H.R. Chuang, E. Postow, An X-band microwave life-detection system. IEEE Transaction on Biomedical Engineering 33, 747-750 (1986)

5. C.G. Bilich, Bio-medical sensing using ultra wideband communications and radar technology, a feasibility study, inIEEE Conference, 1st edn. (, Innsbruck, Austria, 2006), pp. 1-9

6. A. Ruengwaree, A. Ghose, G. Kompa, A novel UWB rugby-ball antenna for near-range microwave radar system. IEEE Transactions on Microwave Theory And Techniques 54, 2774-2779 (2006)

7. X. Huang, B. Chen, H.-L. Cui et al., Theoretical study of sensing targets through the wall using ultra-wideband technology. Proc SPIE 5788, 178-184 (2005)

8. N. Soumya, F. Herbert, B. Mark, Preliminary interferometric images of moving targets obtained using a time-modulated ultra-wide band through-wall penetration radar, inIEEE Radar Conference, 6th edn. (, Atlanta, USA, 2001), pp. 64-69

9. J. Liang, Q. Liang, UWB radar sensor networks detection of targets in foliage using short-time fourier transform, inIEEE ICC Proceedings, 3rd edn. (, Dresden, Germany, 2009), pp. 1-5

10. S.-W. Kim, S.-Y. Kim, S. Nam, Short-time Fourier transform of deeply located tunnel signatures measured by cross-borehole pulse radar. IEEE Geosci Remote Sens Lett 8(3), 493-496 (2011)

11. A.R. Hunt, A wideband imaging radar for through-the-wall surveillance. Proc SPIE 5403, 590-596 (2004)

12. N. Soumya, B. Mark, A moving target detection filter for an ultra-wideband radar, in IEEE Radar Conference, 2nd edn. (, Huntsville, USA, 2003), pp. $147-153$

13. S. Nag, M.A. Barnes, T. Payment et al., An ultra-wideband through-wall radar for detecting the motion of people in real time. Proc SPIE 4744, 48-57 (2002)

14. S. Gauthier, W. Chamma, Surveillance through concrete walls. Proc SPIE 5403, 597-608 (2004)

15. R.M. Narayanan, M.C. Shastry, P.H. Chen et al., Through-the-wall detection of stationary human targets using doppler radar. Progress In Electromagnetics Research B 20, 147-166 (2010)

16. A. Lazaro, D. Girbau, R. Villarino, Analysis of vital signs monitoring using an Ir-Uwb radar. Progress In Electromagnetics Research 100, 265-284 (2010)

doi:10.1186/1687-6180-2012-202

Cite this article as: Zhang et al:: A detecting and identifying method for two stationary human targets using single-channel ultra-wideband radar. EURASIP Journal on Advances in Signal Processing 2012 2012:202. 